EUROPEAN JOURNAL OF PURE AND APPLIED MATHEMATICS

Vol. 13, No. 1, 2020, 1-8

ISSN 1307-5543 - www.ejpam.com

Published by New York Business Global

\title{
Some Closure Operators and Topologies on a Hyper BCK-algebra
}

\author{
Rachel M. Patangan ${ }^{1}$, Sergio R. Canoy, Jr. ${ }^{2, *}$ \\ 1 Department of Applied Mathematics, College of Arts and Sciences, Agusan del Sur State \\ College of Agriculture and Technology, Bunawan, Agusan del Sur, Philippines \\ 2 Department of Mathematics and Statistics, College of Science and Mathematics, \\ Center for Graph Theory, Algebra, and Analysis-PRISM, Mindanao State University- \\ Iligan Institute of Technology, 9200, Iligan City, Philippines
}

\begin{abstract}
Given a hyper BCK-algebra $(H, *, 0)$, we introduce some subsets of $H$ and use them to generate two closure operators on $H$. In this paper, we show that each of the two closure operators on $H$ can be utilized to form a base for some topology on $H$. Moreover, we show that each of the induced topologies coincides with a previously known topology on a hyper BCK-algebra.
\end{abstract}

2020 Mathematics Subject Classifications: 06F35, 03G25

Key Words and Phrases: Hyper BCK-algebra, hyper order, closure operator

\section{Introduction}

The study of BCK-algebras was initiated by Y. Imai and K. Iséki [4] in 1966 as a generalization of the concept of set theoretic difference and propositional calculi. The hyperstructure theory (or multialgebras) was introduced in 1934 by F. Marty [6] at the 8th Congress of Scandinavian Mathematicians in 1934. In [5], Y.B. Jun et al. applied the hyperstructures to BCK-algebras and introduced the notion of a hyper BCK-algebra which is a generalization of a BCK-algebra.

By using the sets $L_{H}(A)$ and $R_{H}(A)$, we introduce the bases $\mathcal{B}_{L}(H)$ and $\mathcal{B}_{R}(H)$ and the induced topologies $\tau_{L}(H)$ and $\tau_{R}(H)$ by these sets, respectively, and investigate their related properties $[7,8]$. In this paper, we present two closure operators on a hyper BCK-algebra and consider the respective topologies they generate. It is shown that these topologies coincide, respectively, with the topologies generated by $\mathcal{B}_{L}(H)$ and $\mathcal{B}_{R}(H)$.

\section{Preliminaries}

A hyper BCK-algebra is a nonempty set $H$ endowed with a hyperoperation "*" and a constant 0 satisfying the following axioms: for all $x, y, z \in H$,

* Corresponding author.

DOI: https://doi.org/10.29020/nybg.ejpam.v13i1.3632

Email addresses: rhapsodistchelar@gmail.com (R. Patangan),

sergio.canoy@g.msuitt.edu.ph (S. Canoy, Jr.) 

(H1) $(x * z) *(y * z) \ll x * y$,
(H3) $x * H \ll x$,
(H2) $(x * y) * z=(x * z) * y$,
(H4) $x \ll y$ and $y \ll x$ imply $x=y$,

where for every $A, B \subseteq H, A \ll B$ if and only if for each $a \in A$, there exists $b \in B$ such that $0 \in a * b$. In particular, for every $x, y \in H, x \ll y$ if and only if $0 \in x * y$. In such case, we call "«" the hyper order in $H$.

Throughout this study, $\left(H_{1}, *_{1}, 0_{1}\right)$ (or simply $\left.H_{1}\right)$ and $\left(H_{2}, *_{2}, 0_{2}\right)$ (or simply $H_{2}$ ) are hyper BCK-algebras.

Let $H$ be a hyper BCK-algebra and $A \subseteq H$. The sets $L_{H}(A)$ and $R_{H}(A)$ are given as follows:

$$
\begin{aligned}
& L_{H}(A):=\{x \in H \mid x \ll a \forall a \in A\}=\{x \in H \mid 0 \in x * a \forall a \in A\} \quad \text { and } \\
& R_{H}(A):=\{x \in H \mid a \ll x \forall a \in A\}=\{x \in H \mid 0 \in a * x \forall a \in A\} .
\end{aligned}
$$

If $A=\{a\}$, we write $L_{H}(\{a\})=L_{H}(a)$ and $R_{H}(\{a\})=R_{H}(a)$.

Let $X$ be a nonempty set and let $\mathscr{P}(X)$ denote the power set of $X$. A mapping $\phi: \mathscr{P}(X) \rightarrow \mathscr{P}(X)$ is called a closure operator on $X$, if for all $A, B \in \mathscr{P}(X)$, the following properties hold [2]:

(i) $A \subseteq \phi(A)$

(ii) $\phi^{2}(A)=\phi(A)$

(iii) $A \subseteq B \Rightarrow \phi(A) \subseteq \phi(B)$.

\section{Known Results}

Proposition 1. [7] Let $H$ be a hyper $B C K$-algebra and $A, B \subseteq H$. Then the following hold:

(i) $R_{H}(\varnothing)=H$.

(ii) $R_{H}(A)=\bigcap_{a \in A} R_{H}(a)$.

(iii) For any $\varnothing \neq A \subseteq H$ such that $A \neq\{0\}, 0 \notin R_{H}(A)$.

(iv) If $A \subseteq B$, then $R_{H}(B) \subseteq R_{H}(A)$.

The next result is generated by Albaracin and Vilela.

Proposition 2. [1] Let $A$ and $B$ be subsets of a hyper BCK-algebra $H$. Then the following hold:

(i) $L_{H}(\varnothing)=H$.

(ii) If $A \subseteq B$, then $L_{H}(B) \subseteq L_{H}(A)$. 
(iii) $L_{H}(A)=\bigcap_{a \in A} L_{H}(a)$.

(iv) For any $A \subseteq H, 0 \in L_{H}(A)$. If $0 \in A$, then $L_{H}(A)=\{0\}$.

Theorem 1. [8] Let $H$ be a hyper BCK-algebra. Then $\mathcal{B}_{L}(H)=\left\{L_{H}(A): A \subseteq H\right\}$ is a basis for some topology on $H$.

Denote by $\tau_{L}(H)$ the topology generated by $\mathcal{B}_{L}(H)$.

Theorem 2. [7] Let $H$ be a hyper BCK-algebra. Then $\mathcal{B}_{R}(H)=\left\{R_{H}(A): \varnothing \neq A \subseteq H\right\}$ is a basis for some topology on $H$.

Denote by $\tau_{R}(H)$ the topology generated by $\mathcal{B}_{R}(H)$.

\section{Topology Induced by $R_{H} L_{H}$}

Let $H$ be any hyper BCK-algebra with $H \neq\{0\}$. By Proposition 1(iii), $0 \notin R_{H}(H)$. By definition of a closure operator, we have the following remark.

Remark 1. $R_{H}: \mathscr{P}(H) \rightarrow \mathscr{P}(H)$ is not a closure operator for every hyper BCK-algebra $H \neq\{0\}$.

Theorem 3. Let $H$ be a hyper $B C K$-algebra and $A, B \subseteq H$. Then the following properties hold:

(i) $A \subseteq R_{H}\left(L_{H}(A)\right)$.

(ii) If $A \subseteq B$, then $R_{H}\left(L_{H}(A)\right) \subseteq R_{H}\left(L_{H}(B)\right)$.

(iii) $\left[R_{H} L_{H}\right]^{2}(A)=R_{H} L_{H}\left[R_{H}\left(L_{H}(A)\right)\right]=R_{H}\left(L_{H}(A)\right)$.

Proof.

(i) Let $A \subseteq H$ and $y \in L_{H}(A)$. Then $y \ll a$ for all $a \in A$. Pick $x \in A$. Then $y \ll x$ for every $y \in L_{H}(A)$. This means that $x \in R_{H}(y)$ for every $y \in L_{H}(A)$. Thus, by Proposition 1(ii), $x \in \bigcap_{y \in L_{H}(A)} R_{H}(y)=R_{H} L_{H}(A)$. Therefore, $A \subseteq R_{H}\left(L_{H}(A)\right)$.

(ii) Let $A, B$ be subsets of $H$. Suppose $A \subseteq B$. By Proposition 2(ii), $L_{H}(B) \subseteq L_{H}(A)$. Thus, by Proposition 1(iv), $R_{H} L_{H}(A) \subseteq R_{H} L_{H}(B)$.

(iii) By (i) and (ii), $R_{H} L_{H}(A) \subseteq\left[R_{H} L_{H}\right]^{2}(A)$. We are left to prove that $\left[R_{H} L_{H}\right]^{2}(A) \subseteq$ $\left(R_{H} L_{H}\right)(A)$. First, we need to show that $L_{H}(A) \subseteq L_{H}\left[R_{H}\left(L_{H}(A)\right)\right]$. Let $x \in$ $R_{H}\left(L_{H}(A)\right)$. Then $y \ll x$ for all $y \in L_{H}(A)$. Choose $z \in L_{H}(A)$. Then $z \ll x$ for every $x \in R_{H}\left(L_{H}(A)\right)$. This implies that $z \in L_{H}(x)$ for every $x \in R_{H}\left(L_{H}(A)\right)$. Hence, by Proposition 2(iii), $z \in \bigcap_{x \in R_{H}\left(L_{H}(A)\right)} L_{H}(x)=L_{H}\left[R_{H}\left(L_{H}(A)\right)\right]$. Consequently, $L_{H}(A) \subseteq L_{H}\left[R_{H}\left(L_{H}(A)\right)\right]$. Therefore, by Proposition 1(iv), $R_{H} L_{H}\left[R_{H}\left(L_{H}(A)\right)\right] \subseteq$ $R_{H}\left(L_{H}(A)\right)$. 
Theorem 4. Let $H$ be a hyper BCK-algebra. The function $R_{H} L_{H}: \mathscr{P}(H) \rightarrow \mathscr{P}(H)$ is a closure operator on $H$.

Proof. Since $H$ is a hyper BCK-algebra, $H \neq \varnothing$. Hence, by the definition of a closure operator and Theorem $3, R_{H} L_{H}$ is a closure operator.

Theorem 5. The family $\mathcal{B}_{R L}(H)=\left\{A: R_{H}\left(L_{H}(A)\right)=A, A \subseteq H\right\}$ is a basis for some topology on $H$.

Proof. Since $R_{H}\left(L_{H}(H)\right) \subseteq H$ and by Theorem 3(i), $R_{H} L_{H}(H)=H$, it follows that $H \in \mathcal{B}_{R L}(H)$. Thus, $\mathcal{B}_{R L}(H) \neq \varnothing$. Let $A, B \in \mathcal{B}_{R L}(H)$ and $x \in A \cap B$. Then by Theorem 3(i), we have $A \cap B \subseteq R_{H}\left(L_{H}(A \cap B)\right)$. Also, since $A \cap B \subseteq A$ and $A \cap B \subseteq B$, $R_{H}\left(L_{H}(A \cap B)\right) \subseteq R_{H}\left(L_{H}(A)\right)$ and $R_{H}\left(L_{H}(A \cap B)\right) \subseteq R_{H}\left(L_{H}(B)\right)$, by Theorem 3(ii). Since $A$ and $B$ are elements of $\mathcal{B}_{R L}(H)$, we have $R_{H}\left(L_{H}(A)\right)=A$ and $R_{H}\left(L_{H}(B)\right)=B$. Consequently, $R_{H}\left(L_{H}(A \cap B)\right) \subseteq A \cap B$. Combining the two set inclusions, we have $R_{H}\left(L_{H}(A \cap B)\right)=A \cap B$, that is, $x \in A \cap B \in \mathcal{B}_{R L}(H)$. Therefore, $\mathcal{B}_{R L}(H)$ is a basis for some topology on $H$.

Denote by $\tau_{R L}(H)$ the topology generated by $\mathcal{B}_{R L}(H)$.

Example 1. Consider the infinite hyper BCK-algebra $(H, *, 0)$ given by Harizavi in [3], where $H=\{0,1,2, \ldots\}$ and "* $*$ is defined as follows:

$$
x * y= \begin{cases}\{0, x\} & \text { if } x \leq y \\ \{x\} & \text { if } x>y\end{cases}
$$

for all $x, y \in H$. Now, let $r \in H$. Then $R_{H}\left(L_{H}(r)\right)=R_{H}(\{0,1,2, \ldots, r\})=\{r, r+$ $1, r+2, \ldots\} \neq\{r\}$. Hence, for any $s \in H,\{s\} \notin \mathcal{B}_{R L}(H)$. Let $\varnothing \neq A \subseteq H$ be a finite set and let $u=\min A$. Since $R_{H}\left(L_{H}(A)\right)=R_{H} L_{H}(u)=R_{H}(\{0,1,2, \ldots, u\})=$ $\{u, u+1, u+2, \ldots\}=R_{H}(u) \neq A$, it follows that for any finite set $A \subseteq H, A \notin \mathcal{B}_{R L}(H)$. Next, suppose that $A \subseteq H$ is an infinite set and let $p=\min A$. Then $R_{H}\left(L_{H}(A)\right)=$ $R_{H}(p)=\{p, p+1, p+2, \ldots\}$. Thus, $A \in \mathcal{B}_{R L}(H)$ if and only if $A=\{p, p+1, p+2, \ldots\}$. Therefore, $\tau_{R L}(H)=\{\varnothing, H\} \cup\{\{p, p+1, p+2, \ldots\}: p \in H\}$.

In the next example, we extend the set $H$ in Example 1 by adjoining to it the set $\left\{\frac{1}{n}: n=2,3, \ldots\right\}$.

Example 2. Consider now the infinite hyper BCK-algebra $(H, \circ, 0)$ given also by Harizavi in [3], where $H=\{0,1,2, \ldots\} \cup\left\{\frac{1}{n}: n=2,3, \ldots\right\}$ and "०" is defined as follows:

$$
x \circ y= \begin{cases}\{0, x\} & \text { if } x \leq y \\ \{x\} & \text { if } x>y\end{cases}
$$

for all $x, y \in H$. For convenience, let $\mathbb{N}=\{1,2, \ldots\}, \mathbb{N}^{0}=\mathbb{N} \cup\{0\}$, and let $J_{k}=\left\{\frac{1}{m}\right.$ : $m$ is a positive integer and $m \geq k\}$. Let $p \in H$. If $p=0$, then $L_{H}(p)=\{0\}$ and $R_{H}\left(L_{H}(p)\right)=H$. If $p \in \mathbb{N}$, then $L_{H}(p)=J_{2} \cup\{0,1,2, \ldots, p\}$. Hence, $R_{H}\left(L_{H}(p)\right)=$ $R_{H}\left(J_{2} \cup\{0,1,2, \ldots, p\}\right)=\{p, p+1, p+2, \ldots\}$. If $p=\frac{1}{n}$ for some $n \in\{2,3, \ldots\}$, then 
$L_{H}(p)=J_{n} \cup\{0\}=\left\{0, \frac{1}{n}, \frac{1}{n+1}, \frac{1}{n+2}, \ldots\right\}$. It follows that $R_{H}\left(L_{H}(p)\right)=R_{H}\left(J_{n} \cup\{0\}\right)=$ $\left\{x \in H: a \leq x\right.$ for all $\left.a \in\left(J_{n} \cup\{0\}\right)\right\}=\mathbb{N} \cup\left\{\frac{1}{2}, \frac{1}{3}, \ldots, \frac{1}{n}\right\}$.

Now let $\varnothing \neq A \subseteq H$. If $0 \in A$, then $L_{H}(A)=\{0\}$ and $R_{H}\left(L_{H}(A)\right)=H$. Thus, $A \in$ $\mathcal{B}_{R L}(H)$ if and only if $A=H$. Next, suppose that $0 \notin A$. Suppose first that $A \cap J_{2} \neq \varnothing$. Suppose that $A \cap J_{2}$ is infinite and suppose further that there exists $z \in L_{H}(A)$. Then $z \leq a$ for all $a \in A$. It follows that $z \leq b$ for all $b \in A \cap J_{2}$, contrary to the assumption that $A \cap J_{2}$ is infinite. Thus, $L_{H}(A)=\varnothing$. Next, suppose that $A \cap J_{2}$ is finite and let $\frac{1}{q}=\min \left(A \cap J_{2}\right)$. Then $L_{H}(A)=J_{q} \cup\{0\}$. Hence, $R_{H}\left(L_{H}(A)\right)=\mathbb{N} \cup\left\{\frac{1}{2}, \frac{1}{3}, \ldots, \frac{1}{q}\right\}$. Therefore, $A \in \mathcal{B}_{R L}(H)$ if and only if $A=\mathbb{N} \cup\left\{\frac{1}{2}, \frac{1}{3}, \ldots, \frac{1}{q}\right\}$. Suppose now that $A \cap J_{2}=\varnothing$. Then $L_{H}(A)=$ $J_{2} \cup\{0,1,2, \ldots, r\}$, where $r=\min A$. Thus, $R_{H}\left(L_{H}(A)\right)=R_{H}\left(J_{2} \cup\{0,1,2, \ldots, r\}\right)=$ $\{r, r+1, r+2, \ldots\}$. Thus, $A \in \mathcal{B}_{R L}(H)$ if and only if $A=\{r, r+1, r+2, \ldots\}$. Therefore, $\tau_{R L}(H)=\{\varnothing, H\} \cup\{\{p, p+1, p+2, \ldots\}: p \in \mathbb{N}\} \cup\left\{\mathbb{N} \cup\left\{\frac{1}{2}, \frac{1}{3}, \ldots, \frac{1}{k}\right\}: k=2,3, \ldots\right\}$.

It can be observed that the topology $\tau_{R L}(H)$ in Example 1 coincides with the topology $\tau_{R}(H)$ in Example 2.5 [7]. In fact, the next result shows that this equality is true for any hyper BCK-algebra $H$.

Theorem 6. Let $H$ be a hyper BCK-algebra. Then the topology $\tau_{R L}(H)$ coincides with the topology $\tau_{R}(H)$.

Proof. By Theorem 5, a basis for $\tau_{R L}(H)$ is the family $\mathcal{B}_{R L}(H)=\left\{A: R_{H} L_{H}(A)=\right.$ $A, A \subseteq H\}$ while a basis for $\tau_{R}(H)$ is the family $\mathcal{B}_{R}(H)=\left\{R_{H}(A): \varnothing \neq A \subseteq H\right\}$. Let $A \in \mathcal{B}_{R L}(H)$. Then $R_{H} L_{H}(A)=A$. Set $D=L_{H}(A)$. Then $R_{H}(D)=A \in \mathcal{B}_{R}(H)$, showing that $\mathcal{B}_{R L}(H) \subseteq \mathcal{B}_{R}(H)$. Next, let $U \in \mathcal{B}_{R}(H)$. Then there exists $B \subseteq H$ such that $R_{H}(B)=U$. This implies that for every $u \in U, b \ll u$ for all $b \in B$. Hence, $B \subseteq L_{H}(U)$. By Proposition 1(iv), $R_{H} L_{H}(U) \subseteq R_{H}(B)=U$. Also, by Theorem 3(i), we have $U \subseteq R_{H} L_{H}(U)$. Thus, $U=R_{H} L_{H}(U) \in \mathcal{B}_{R L}(H)$, it shows that $\mathcal{B}_{R}(H) \subseteq \mathcal{B}_{R L}(H)$. Therefore, $\mathcal{B}_{R L}(H)=\mathcal{B}_{R}(H)$. Accordingly, $\tau_{R L}(H)=\tau_{R}(H)$.

\section{Topology Induced by $L_{H} R_{H}$}

Let $H$ be any hyper BCK-algebra with $H \neq\{0\}$. By Proposition 2(iv), $L_{H}(H)=\{0\}$. By definition of a closure operator, we obtain the following remark.

Remark 2. $L_{H}: \mathscr{P}(H) \rightarrow \mathscr{P}(H)$ is not a closure operator for every hyper BCK-algebra $H \neq\{0\}$.

Theorem 7. Let $A, B$ be subsets of a hyper BCK-algebra $H$. Then the following properties hold:

(i) $A \subseteq L_{H} R_{H}(A)$.

(ii) If $A \subseteq B$, then $L_{H}\left(R_{H}(A)\right) \subseteq L_{H}\left(R_{H}(B)\right)$.

(iii) $\left[L_{H} R_{H}\right]^{2}(A)=L_{H} R_{H}\left[L_{H}\left(R_{H}(A)\right)\right]=L_{H}\left(R_{H}(A)\right)$. 
Proof.

(i) Let $A \subseteq H$ and $x \in R_{H}(A)$. Then $a \ll x$ for all $a \in A$. Select $b \in A$. Then $b \ll x$ for every $x \in R_{H}(A)$. This means that $b \in L_{H}(x)$ for every $x \in R_{H}(A)$. Hence, $b \in L_{H}\left(R_{H}(A)\right)$. Therefore, $A \subseteq L_{H}\left(R_{H}(A)\right)$.

(ii) Let $A, B \subseteq H$ such that $A \subseteq B$. By Proposition $1\left(\right.$ iv), $R_{H}(B) \subseteq R_{H}(A)$. Thus, by Proposition $2(\mathrm{ii}), L_{H}\left(R_{H}(A)\right) \subseteq L_{H}\left(R_{H}(B)\right)$.

(iii) By (i) and (ii), $L_{H}\left(R_{H}(A)\right) \subseteq\left[L_{H} R_{H}\right]^{2}(A)$. We are left to prove that $\left[L_{H} R_{H}\right]^{2}(A) \subseteq$ $L_{H}\left(R_{H}(A)\right)$. First, we need to show that $R_{H}(A) \subseteq R_{H}\left[L_{H}\left(R_{H}(A)\right)\right]$. Let $x \in$ $L_{H}\left(R_{H}(A)\right)$. Then $x \ll y$ for all $y \in R_{H}(A)$. Take $z \in R_{H}(A)$. It follows that $x \ll z$ for every $x \in L_{H}\left(R_{H}(A)\right)$. This implies that $z \in R_{H}(x)$ for each $x \in L_{H}\left(R_{H}(A)\right)$. Hence, $z \in R_{H}\left[L_{H}\left(R_{H}(A)\right)\right]$. This shows that $R_{H}(A) \subseteq R_{H}\left[L_{H}\left(R_{H}(A)\right)\right]$. Thus, by Proposition 2(ii), $L_{H} R_{H}\left[L_{H}\left(R_{H}(A)\right)\right] \subseteq L_{H}\left(R_{H}(A)\right)$. By combining the two set inclusions, we have $L_{H} R_{H}\left[L_{H}\left(R_{H}(A)\right)\right]=L_{H}\left(R_{H}(A)\right)$.

Theorem 8. Let $H$ be a hyper BCK-algebra. The function $L_{H} R_{H}: \mathscr{P}(H) \rightarrow \mathscr{P}(H)$ is a closure operator on $H$.

Proof. Since $H$ is a hyper BCK-algebra, $H \neq \varnothing$. Then by the definition of a closure operator and Theorem $7, L_{H} R_{H}$ is a closure operator.

Theorem 9. The family $\mathcal{B}_{L R}(H)=\left\{A: L_{H} R_{H}(A)=A, \varnothing \neq A \subseteq H\right\}$ is a basis for some topology on $H$.

Proof. Since $L_{H} R_{H}(H) \subseteq H$ and by Theorem $7(\mathrm{i})$, we have $L_{H} R_{H}(H)=H$ and so, $H \in \mathcal{B}_{L R}(H)$. Thus, $\mathcal{B}_{L R}(H) \neq \varnothing$. Next, let $A, B \in \mathcal{B}_{L R}(H)$ and $x \in A \cap B$. Then by Theorem $7(\mathrm{i})$, we have $A \cap B \subseteq L_{H} R_{H}(A \cap B)$. Also, since $A \cap B \subseteq A$ and $A \cap B \subseteq B, L_{H} R_{H}(A \cap B) \subseteq L_{H} R_{H}(A)$ and $L_{H} R_{H}(A \cap B) \subseteq L_{H} R_{H}(B)$ by Theorem 7(ii). Since $A, B \in \mathcal{B}_{L R}(H)$, we have $L_{H} R_{H}(A)=A$ and $L_{H} R_{H}(B)=B$. Hence, $L_{H} R_{H}(A \cap B) \subseteq A \cap B$. Accordingly, $L_{H} R_{H}(A \cap B)=A \cap B \in \mathcal{B}_{L R}(H)$. This shows that $\mathcal{B}_{L R}(H)$ is a basis for some topology on $H$.

Denote by $\tau_{L R}(H)$ the topology generated by $\mathcal{B}_{L R}(H)$.

Example 3. Consider the hyper BCK-algebra $(H, *, 0)$ in Example 1. Let $n \in H$. Then $L_{H} R_{H}(n)=L_{H}(\{n, n+1, n+2, \ldots\})=\{0,1,2, \ldots, n\} \neq\{n\}$. Hence, for any $m \in H$, $\{m\} \notin \mathcal{B}_{L R}(H)$. Let $A$ be any infinite subset of $H$. Then $L_{H} R_{H}(A)=L_{H}(\varnothing)=H \neq A$. It follows that $A \notin \mathcal{B}_{L R}(H)$. Now, suppose that $A$ is any finite set of $H$ such that $A=$ $\{0,1,2, \ldots, r\}$. Then $L_{H} R_{H}(A)=L_{H}(\{r, r+1, r+2, \ldots\})=\{0,1,2, \ldots, r\}=A$. Thus, $\{0,1,2, \ldots, r\} \in \mathcal{B}_{L R}(H)$. Now, for any finite set $B \neq A, L_{H} R_{H}(B) \neq B$. Therefore, $\tau_{L R}(H)=\{\varnothing, H\} \cup\{\{0,1,2, \ldots, r\}: r \in H\}$.

Example 4. Consider the infinite hyper BCK-algebra $(H, \circ, 0)$ given in Example 2. Again, for convenience, let $\mathbb{N}=\{1,2, \ldots\}, \mathbb{N}^{0}=\mathbb{N} \cup\{0\}$, and let $J_{k}=\left\{\frac{1}{m}: m\right.$ is a positive integer 
and $m \geq k\}$. Let $p \in H$. If $p=0$, then $R_{H}(0)=\{x \in H: 0 \leq x\}=H$ and $L_{H}\left(R_{H}(0)\right)=$ $L_{H}(H)=\{0\}$. It follows that $\{0\} \in \mathcal{B}_{L R}(H)$. Let $p \in \mathbb{N}$. Then $R_{H}(p)=\{x \in H$ : $p \leq x\}=\{p, p+1, p+2, \ldots\}$. It follows that $L_{H}\left(R_{H}(p)\right)=L_{H}(\{p, p+1, p+2, \ldots\})=$ $J_{2} \cup\{0,1,2, \ldots, p\}$. If $p=\frac{1}{n}$ for some $n \in\{2,3, \ldots\}$, then $R_{H}(p)=\left\{x \in H: \frac{1}{n} \leq x\right\}=\mathbb{N} \cup$ $\left\{\frac{1}{2}, \frac{1}{3}, \ldots, \frac{1}{n}\right\}$. Hence, $L_{H}\left(R_{H}(p)\right)=L_{H}\left(\mathbb{N} \cup\left\{\frac{1}{2}, \frac{1}{3}, \ldots, \frac{1}{n}\right\}\right)=J_{n} \cup\{0\}=\left\{0, \frac{1}{n}, \frac{1}{n+1}, \frac{1}{n+2}, \ldots\right\}$.

Next, let $\varnothing \neq A \subseteq H$ with $A \neq\{0\}$. Suppose first that $A \cap \mathbb{N} \neq \varnothing$. If $A \cap \mathbb{N}^{0}$ is infinite, then $R_{H}(A)=\varnothing$ and $L_{H}\left(R_{H}(A)\right)=H$. Suppose $A \cap \mathbb{N}$ is finite and let $s=\max (A \cap \mathbb{N})$. Then $R_{H}(A)=R_{H}(s)$ and $L_{H}\left(R_{H}(A)\right)=J_{2} \cup\{0,1,2, \ldots, s\}$. Hence, $A \in \mathcal{B}_{L R}(H)$ if and only if $A=H$ or $A=J_{2} \cup\{0,1,2, \ldots, s\}$.

Suppose now that $A \cap \mathbb{N}=\varnothing$. Since $A \neq\{0\}, A \cap J_{2} \neq \varnothing$. Let $d=\min \{k \in\{2,3, \ldots\}$ : $\left.\frac{1}{k} \in A\right\}$. Then $R_{H}(A)=R_{H}\left(\frac{1}{d}\right)=\mathbb{N} \cup\left\{\frac{1}{2}, \frac{1}{3}, \ldots, \frac{1}{d}\right\}$ and $L_{H}\left(R_{H}(A)\right)=J_{d} \cup\{0\}=$ $\left\{0, \frac{1}{d}, \frac{1}{d+1}, \frac{1}{d+2}, \ldots\right\}$. Consequently, $A \in \mathcal{B}_{L R}(H)$ if and only if $A=\left\{0, \frac{1}{d}, \frac{1}{d+1}, \frac{1}{d+2}, \ldots\right\}$.

Therefore, $\tau_{L R}(H)=\{\varnothing,\{0\}, H\} \cup\left\{J_{2} \cup\{0,1,2, \ldots, p\}: p \in H\right\} \cup\left\{\left\{0, \frac{1}{k}, \frac{1}{k+1}, \frac{1}{k+2}, \ldots\right\}:\right.$ $k=2,3, \ldots\}$.

It can be seen that the topology $\tau_{L R}(H)$ generated in Example 3 is equal to the topology $\tau_{L}(H)$ generated in Example 2.5 [8]. The next result says that the equality of these topologies holds for any hyper BCK-algebra $H$.

Theorem 10. Let $H$ be a hyper BCK-algebra. The the topology $\tau_{L R}(H)$ coincides with the topology $\tau_{L}(H)$.

Proof. By Theorem 9, a basis for $\tau_{L R}(H)$ is given by $\mathcal{B}_{L R}(H)=\left\{A: L_{H} R_{H}(A)=\right.$ $A, \varnothing \neq A \subseteq H\}$ while a basis for $\tau_{L}(H)$ is the family $\mathcal{B}_{L}(H)=\left\{L_{H}(A): \varnothing \neq A \subseteq H\right\}$. Let $A \in \mathcal{B}_{L R}(H)$. Then $L_{H} R_{H}(A)=A$. Put $B=R_{H}(A) \subseteq H$. Then $L_{H}(B)=A \in$ $\mathcal{B}_{L}(H)$, showing that $\mathcal{B}_{L R}(H) \subseteq \mathcal{B}_{L}(H)$. Next, let $V \in \mathcal{B}_{L}(H)$. Then there exists $D \subseteq H$ such that $L_{H}(D)=V$. Now, for every $v \in V, v \ll d$ for all $d \in D$. Thus, $D \subseteq R_{H}(V)$. By Proposition 2(ii), $L_{H} R_{H}(V) \subseteq L_{H}(D)=V$. By Theorem $7(\mathrm{i})$, we have $V \subseteq L_{H} R_{H}(V)$. By combining the two set inclusions, we obtain $V=L_{H} R_{H}(V) \in \mathcal{B}_{L R}(H)$. Accordingly, $\mathcal{B}_{L R}(H)=\mathcal{B}_{L}(H)$, showing that $\tau_{L R}(H)=\tau_{L}(H)$.

Conclusion Right and left applications of a hyper order associated with a hyper BCKalgebra can give rise to two closure operators which, in turn, can be used to generate bases for some topologies on the given hyper structure. The two induced topologies turn out to coincide, respectively, with some previously known topologies on this hyper BCK-algebra.

\section{Acknowledgements}

The authors would like to thank the referees for their invaluable comments and corrections which led to the improvement of the manuscript. This research is funded by ASSCAT and MSU-Iligan Institute of Technology. 


\section{References}

[1] J Albaracin and J Vilela. Zero Divisor Graph of Finite Hyper BCK-algebra involving hyperatoms. Far East Journal Mathematical Sciences, 103(4):743-755, 2018.

[2] S Burris and H Sankappanavar. A course in Universal Algebra. Springer-Verlag, New York., Heidelbery, Berlin, 1981.

[3] H Harizavi. On Direct Sum of Branches in Hyper BCK-algebras. Iranian Journal of Mathematical Sciences and Informatics, 11(2):43-55, 2016.

[4] Y Imai and K Iséki. On Axiom systems of Propositional Calculi XIV. Proc. Japan Academy, 42:19-22, 1966.

[5] Y Jun M Zahedi, X Xin and R Borzooei. On Hyper BCK-algebras. Italian Journal of Pure and Applied Mathematics, 8:127-136, 2000.

[6] F Marty. Sun une Generalization da la Notion de Group. Stockholm: 8th Congress Math. Scandinaves, pages 45-49, 1934.

[7] R Patangan and S Canoy Jr. A Topology on a Hyper BCK-algebra. JP Journal of Algebra, Number Theory and Applications, 40:787-797, 2018.

[8] R Patangan and S Canoy Jr. A Topology on a Hyper BCK-algebra via Left Application of a Hyper Order. JP Journal of Algebra, Number Theory and Applications, 40:321$332,2018$. 